\title{
Robust Detection of Singularities in Vector Fields
}

H. Bhatia, A. Gyulassy, H. Wang, P. T. Bremer, V. Pascucci

October 18, 2013

TopolnVis

Davis, CA, United States

March 4, 2013 through March 6, 2013 
This document was prepared as an account of work sponsored by an agency of the United States government. Neither the United States government nor Lawrence Livermore National Security, LLC, nor any of their employees makes any warranty, expressed or implied, or assumes any legal liability or responsibility for the accuracy, completeness, or usefulness of any information, apparatus, product, or process disclosed, or represents that its use would not infringe privately owned rights. Reference herein to any specific commercial product, process, or service by trade name, trademark, manufacturer, or otherwise does not necessarily constitute or imply its endorsement, recommendation, or favoring by the United States government or Lawrence Livermore National Security, LLC. The views and opinions of authors expressed herein do not necessarily state or reflect those of the United States government or Lawrence Livermore National Security, LLC, and shall not be used for advertising or product endorsement purposes. 


\title{
Robust Detection of Singularities in Vector Fields
}

\author{
Harsh Bhatia, Attila Gyulassy, Hao Wang, Peer-Timo Bremer, and Valerio Pascucci
}

\begin{abstract}
Recent advances in computational science enable the creation of massive datasets of ever increasing resolution and complexity. Dealing effectively with such data requires new analysis techniques that are provably robust and that generate reproducible results on any machine. In this context, combinatorial methods become particularly attractive, as they are not sensitive to numerical instabilities or the details of a particular implementation. We introduce a robust method for detecting singularities in vector fields. We establish, in combinatorial terms, necessary and sufficient conditions for the existence of a critical point in a cell of a simplicial mesh for a large class of interpolation functions. These conditions are entirely local and lead to a provably consistent and practical algorithm to identify cells containing singularities.
\end{abstract}

\section{Introduction}

Vector fields, which are either acquired from real-world experiments or generated by computer simulations, are ubiquitous in scientific research. In recent years, the increase in computational power coupled with the ability to simulate ever more complex data has greatly increased the need for the automatic analysis of large-scale vector fields. To handle the complexity and size of modern simulations, flexible, multi-scale methods are needed. These techniques must be able to define features at different levels of resolution, remove noise, and most importantly be computationally robust and consistent with the mathematical theory of vector fields. Stateof-the-art topological techniques fulfill many of these requirements by defining features based on the global behavior of streamlines, which are the paths parallel to the direction of flow of a fluid at a given instant, and by providing feature-based simpli-

Harsh Bhatia, Attila Gyulassy, Peer-Timo Bremer, and Valerio Pascucci

SCI Institute, University of Utah, USA. e-mail: \{hbhatia,jediati,ptbremer,pascucci\}@ sci.utah.edu.

Harsh Bhatia and Peer-Timo Bremer

Lawrence Livermore National Laboratory, CA, USA. e-mail: \{bhatia4,bremer5\}@1lnl.gov. 
fication. For example, topological techniques for 2D vector fields use singularities - points where the field is zero, such as sources, sinks, saddles, etc., together with separatrices - the streamlines of a saddle, and limit cycles - the streamlines which wrap back onto themselves, to decompose the vector field into regions of similar flow behavior. Such decompositions are then used to analyze, visualize [21, 24], simplify [29, 31], and compress [23] vector fields.

However, the majority of analysis and visualization techniques are based on the direct application of the theory of smooth vector fields to sampled flows, ignoring the fact that real numbers are replaced by finite-precision floating point arithmetic. As a result, such numerical approaches may lead to the lack of consistency, meaning that the fundamental laws of smooth mathematical theory may not be preserved in practical applications. One type of inconsistency is the topological inconsistency, e.g. intersection of streamlines, violation of the Poincaré-Hopf theorem, etc. In order to obtain consistent results, the analysis must have numerical robustness, meaning that it is independent of the underlying machine and/or floating point standards. Numerical instabilities may produce topological structures which are incorrect or inconsistent, hence questioning the fidelity of any subsequent analysis.

In several application areas, singularities of scalar fields have been shown to correspond to features of interest. For example, Laney et al. [9] use the gravitational potential on an envelope surface to indicate mixing structures in a Rayleigh-Taylor simulation. Mascarenhas et al. [15] and Bremer et al. [2] use extrema in fields derived from different combustion simulations to count regions of flame extinction and strong burning, respectively. Robust singularity detection in vector fields promises similar results for new applications such as turbulent flow analysis. As a result, the focus of the current work is a robust technique for the detection of singularities, which allows for consistent analysis.

Real-world data is most often available as discrete samples at the vertices of a mesh, and a continuous function is recovered through interpolation of these values. The particular case of piecewise-linear (PL) interpolants has been studied extensively in the context of identification and representation of singularities [8, 27, 29, 31]. The simplicity of linear interpolation makes PL vector fields preferred in many applications. However, even with this simple interpolant, robust identification of singularities remains a challenge, as in the case of many non-

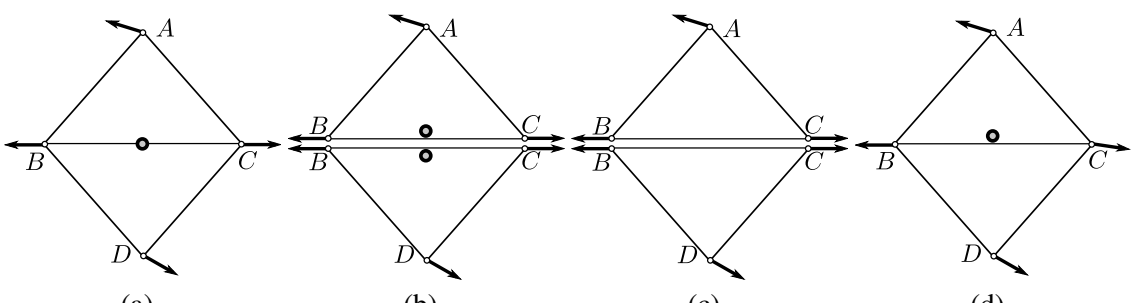

(a)

(b)

(c)

(d)

Fig. 1 (a) When $\mathbf{v}_{B}$ and $\mathbf{v}_{C}$ are anti-parallel, a singularity exists on the edge $B C$. Numerical techniques for identifying triangles containing critical points might test positive for (b) both $A B C$ and $B D C$, or (c) neither of them. (d) A practical implementation of our combinatorial technique uses Simulation of Simplicity [5] to consistently determine the triangle containing the critical point. 
combinatorial geometric algorithms [22, 32]. For example, linear interpolation on a simplicial domain may admit singularities on the boundaries of simplices - an unstable configuration highly sensitive to numerical perturbation. Fig. 1 shows how non-combinatorial methods may yield false positives or false negatives in these configurations, leading to inconsistent analysis. Furthermore, a large number of fluid simulations impose no-slip boundary conditions, meaning that the vectors at the boundary are zero. These zero-vectors are the artifacts of the boundary/simulation rather than singularities of the field. While it is possible to filter them out of the critical point identification, it requires an explicit manual intervention, on top of a priori knowledge of the boundary conditions.

In contrast to numerical approaches, some combinatorial alternatives [18, 19] have been proposed to extract the topological structure from vector fields. Such techniques typically convert the input data into a discrete form to obtain numerical robustness, however, they are severely limited since it is not known how well do these discrete techniques approximate the field.

To address these problems, new combinatorial techniques are required which are guaranteed to detect all topological structures in a manner consistent with the fundamental principles of vector fields. To this end, our contributions are:

- We prove necessary and sufficient conditions for the existence of singularities within cells of a simplicial mesh for a broad class of interpolation functions.

- We show how to turn the necessary and sufficient conditions for the existence of singularities into a combinatorial algorithmic approach.

\section{Related Work}

The topological skeleton of vector fields introduced by Helman and Hesselink [8] is of special interest to many researchers. It consists of important features of the field such as singularities, saddle separatrices, and limit cycles. Thus, the identification and classification of these features is an integral part of the analysis and visualization of vector fields. However, most of the early attempts at identification of singularities were based on numerical analysis. For example, isolated non-degenerate singularities were identified using numerically integrated tangent curves (streamlines) and classified based on eigenvalue analysis [8]. Lavin et al. [10] and Batra and Hesselink [1] extract singularities in a PL vector field $\mathbf{V}=\mathbf{A x}+\mathbf{o}$ by numerically solving the system $\mathbf{A x}=\mathbf{0}$ for each cell in a triangulated domain.

The detection of singularities has also been extended to higher-order singularities [20]. Tricoche et al. [27] analyze higher-order singularities in 2D by partitioning the neighborhood of the singularity into sectors of different flow behavior. The topological analysis of higher order singularities provides a foundation for the design and simplification of vector fields. Tricoche et al. [28] simplify the topology of vector fields by merging clustered singularities within a convex polygon into higher order singularities. These ideas have been extended to 3D by Weinkauf et al. [30, 31]. It is more challenging to identify singularities in nonlinear vector fields. Li et al. [11] 
subdivide the simplicial mesh and compute the vector field by side-vertex interpolation in polar coordinates. Singularities are then ensured to be located at the vertices.

In general, detection of singularities can be reformulated as solving nonlinear systems of equations. The Newton-Raphson method and Broyden's method can be used to solve such systems. However, techniques aimed at solving generic nonlinear systems are sensitive to perturbation and not guaranteed to find all the solutions. For multivariate rational splines, Elber and Kim [6] apply the bisection method to localize the potential regions containing roots. However, computational complexity is a major concern of their method.

Consistency and robustness are particularly desired when computing the topological skeleton of a vector field. A number of techniques have been proposed to extract it in a stable and efficient manner. Such techniques range from deriving some properties from the original vector field, and basing the extraction on those properties; to converting the vector field into a simpler combinatorial form which makes the extraction more robust.

For example, Polthier and Preuß [17] detect singularities as the extrema of the rotation-free and divergence-free potentials obtained from the discrete HelmholtzHodge decomposition of the vector field. This method, however, only works for piecewise-constant $(\mathrm{PC})$ vector fields. Chen et al. propose the Entity Connection Graph (ECG) [3] and the Morse Connection Graph (MCG) [4] as the topological representation of PL vector fields. However, both ECG and MCG do not represent higher order features of the field. On the other hand, Reininghaus et al. [18, 19] construct a combinatorial vector field. While using their combinatorial fields enables the extraction of a consistent topological structure, it is unclear how close the resulting combinatorial field is to the original field. By comparison, this work proposes a robust and consistent combinatorial identification of singularities by working directly on the input vector field.

In addition to the techniques discussed above, the notion of Poincaré index also inspires combinatorial approaches to detect critical points. In this context, Garth et al. [7] propose a method to detect and track singularities in time-dependent vector fields by ensuring the Poincaré index is always preserved. Other techniques ensuring the validity of Poincare index include the works of Mann and Raywood [14] and Trioche et al. [25, 26].

\section{Foundations}

Let $D$ be a bounded, open subset of $\mathbb{R}^{n}$. The closure and the boundary of $D$ are denoted by $\bar{D}$ and $\partial D$ respectively. A point $\mathbf{x} \in \mathbb{R}^{n}$ is denoted as $\mathbf{x}=\left(\mathbf{x}_{0}, \ldots, \mathbf{x}_{n-1}\right)$, and has an L-infinity norm $|\mathbf{x}|=\max \left\{\left|\mathbf{x}_{i}\right| ; i=0, \cdots, n-1\right\} . C(\bar{D})$ denotes the class of continuous functions $\phi \in C(\bar{D})$, such that $\phi(\mathbf{x}): \bar{D} \rightarrow \mathbb{R}^{n}$ with the norm $\|\phi\|=$ $\sup _{\mathbf{x} \in D}|\phi(\mathbf{x})| . C^{1}(\bar{D})$ is a subset of $C(\bar{D})$ such that $\phi \in C^{1}(\bar{D})$ has continuous firstorder partial derivatives. Let $\mathbf{p}=\phi(\mathbf{x})=\left(\phi_{0}(\mathbf{x}), \cdots, \phi_{n-1}(\mathbf{x})\right)$, then, the Jacobian matrix $\mathbf{J}$ of $\phi$ is given as $\mathbf{J} \phi(\mathbf{x})=\left[\nabla \phi_{0}, \cdots, \nabla \phi_{n-1}\right]^{T}$. For $\phi \in C^{1}(\bar{D}), \mathbf{p}=\phi(\mathbf{x})$ is called a degenerate value of $\phi$ if there exists $\mathbf{x} \in \bar{D}$ such that $\operatorname{det}(\mathbf{J} \phi(\mathbf{x}))=\mathbf{0}$, otherwise $\mathbf{p}$ is a regular value of $\phi$. 


\subsection{Degree Theory}

The proposed critical point detection technique requires results relating the existence of certain values in the image of a function to the span of the image. We are interested in a particular class of functions $\phi: \mathbb{R}^{n} \rightarrow \mathbb{R}^{n}$ for which the pre-image of a non-degenerate value $\mathbf{p}$ is a finite set of points. Therefore, we utilize the Brouwer degree as a tool for determining whether or not a particular value exists inside the image of a simplex. The following definitions have been made by Lloyd [12].

For a bounded, open subset $D \subset \mathbb{R}^{n}$, and a continuous function $\phi \in C(\bar{D})$, the Brouwer degree of $\phi$ in $D$ with respect to the value $\mathbf{p}$, where $\mathbf{p} \notin \phi(\partial D)$ is defined as follows:

Definition 1 (Brouwer Degree for $C^{1}(\bar{D})$ and regular value p). If $\phi \in C^{1}(\bar{D})$ and $\mathbf{p}$ is a regular value of $\phi$, then

$$
\operatorname{deg}(\phi, D, \mathbf{p})=\sum_{\mathbf{x} \in \phi^{-1}(\mathbf{p})} \operatorname{sign}(\operatorname{det}(\mathbf{J} \phi(\mathbf{x})))
$$

An intuition behind the concept of Brouwer degree is illustrated in Fig. 2(a). It is essentially the count of the net crossings of $\mathbf{p}$ by the image of $\bar{D}$ under $\phi$. The above definition is limited to regular values $\mathbf{p}$ only. Not all values in a sampled function reconstructed through interpolation are regular, so our definition must encompass degenerate values as well.

Definition 2 (Brouwer Degree for $C^{1}(\bar{D})$ and degenerate value p). If $\phi \in C^{1}(\bar{D})$ and $\mathbf{p}$ is a degenerate value of $\phi$, then

$$
\operatorname{deg}(\phi, D, \mathbf{p})=\operatorname{deg}\left(\phi, D, \mathbf{p}_{1}\right)
$$

where $\mathbf{p}_{1}$ is any regular value such that $\left|\mathbf{p}-\mathbf{p}_{1}\right|<\operatorname{dist}(\mathbf{p}, \phi(\partial D))$.

The existence of a non-degenerate value in every neighborhood of $\mathbf{p}$ is guaranteed by Sard's Theorem [12]. Similarly, we would like to extend this definition to functions that are continuous, but not necessarily in $C^{1}(\bar{D})$.

Definition 3 (Brouwer Degree for $C(\bar{D})$ ). If $\phi \in C(\bar{D})$, then

$$
\operatorname{deg}(\phi, D, \mathbf{p})=\operatorname{deg}\left(\phi_{1}, D, \mathbf{p}\right)
$$

where $\phi_{1}$ is any function in $C^{1}(\bar{D})$ such that for any $\mathbf{x} \in \bar{D},\left|\phi(\mathbf{x})-\phi_{1}(\mathbf{x})\right|<$ $\operatorname{dist}(\mathbf{p}, \phi(\partial D))$.

Basically, we can find a function $\phi_{1}$ that is "close" to $\phi$ and has continuous derivatives, and define the Brouwer degree with respect to this function.

Using the Brouwer degree, the net number of crossings of $\mathbf{p}$ by the image of $\bar{D}$ can be counted. If this number is nonzero, then there exists at least one $\mathbf{x}$ such that $\phi(\mathbf{x})=\mathbf{p}$. This leads to the following theorems: 


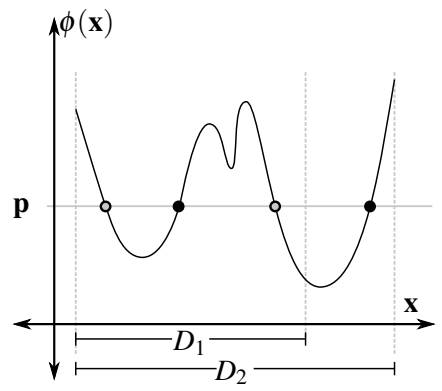

(a)

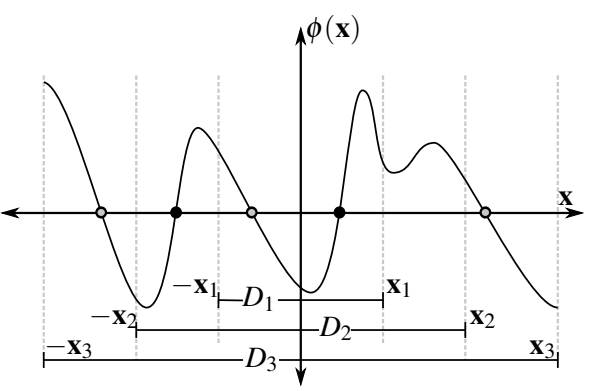

(b)

Fig. 2 The Brouwer degree counts the net number of times $\mathbf{p}$ is crossed by the image of $\bar{D}$ under $\phi$. The "positive" $(\operatorname{det}(\mathbf{J} \phi(\mathbf{x}))>0)$ and "negative" $(\operatorname{det}(\mathbf{J} \phi(\mathbf{x}))<0)$ crossings are shown as solid and hollow dots respectively. (a) For the open and bounded sets $D_{1}$ and $D_{2}$, the Brouwer degree of $\phi(\mathbf{x})$ with respect to the value $\mathbf{p}$ is -1 and 0 respectively. (b) When the sets $D_{i}$ are symmetric, Theorem 2 guarantees that if the values on the boundary have different signs, then the Brouwer degree with respect to $\mathbf{0}$ is odd, as is the case for $D_{2}$ and $D_{3}$.

Theorem 1 (Kronecker's Existence Theorem). If $\operatorname{deg}(\phi, D, \mathbf{p}) \neq 0$, then the equation $\phi(\mathbf{x})=\mathbf{p}$ has at least one solution in $D$.

Theorem 2. Let $D$ be a bounded, open, symmetric subset of $\mathbb{R}^{n}$ containing the origin. If $\phi: \bar{D} \rightarrow \mathbb{R}^{n}$ is continuous, $\mathbf{0} \notin \phi(\partial D)$, and for all $\mathbf{x} \in \partial D$

$$
\frac{\phi(\mathbf{x})}{|\phi(\mathbf{x})|} \neq \frac{\phi(-\mathbf{x})}{|\phi(-\mathbf{x})|}
$$

then $\operatorname{deg}(\phi, D, \mathbf{0})$ is an odd number [12].

Intuitively, Theorem 2 ensures that $\phi$ crosses $\phi(\mathbf{x})=0$ at least once if no antipodal vectors of $\phi$ are parallel, as can be seen in Fig. 2(b).

\subsection{Sampled Vector Fields}

The proposed technique addresses the detection of singularities for interpolated vector fields, where vectors are defined on the vertices of a simplicial complex and then interpolated on the interior of simplices.

A $k$-simplex, $\mathbb{S}^{k}$, is the convex hull of $k+1$ affinely-independent vertices, such that $\mathbb{S}^{k}=\left\{\mathbf{x}_{i}\right\}, \mathbf{x}_{i} \in \mathbb{R}^{n}, 0 \leq i \leq k \leq n$. A simplex $\mathbb{S}^{l}$ is called a $l$-face of $\mathbb{S}^{k}$ for $l \leq k$ if $\mathbb{S}^{l} \subseteq \mathbb{S}^{k}$, and a proper $l$-face of $\mathbb{S}^{k}$ for $l<k$ if $\mathbb{S}^{l} \subset \mathbb{S}^{k}$. A proper $k-1$-face $\mathbb{S}_{j}^{k-1}$ of $\mathbb{S}^{k}$ is called its facet if $\mathbb{S}_{j}^{k-1}=\left\{\mathbf{x}_{i}\right\}, \mathbf{x}_{i} \subset \mathbb{S}^{k}, 0 \leq i \leq k \leq n, i \neq j$, i.e. it is constructed by removing vertex $x_{j}$ from $\mathbb{S}^{k}$. If $\mathbb{S}^{l}$ is a (proper) face of $\mathbb{S}^{k}$, then $\mathbb{S}^{k}$ is called a (proper) coface of $\mathbb{S}^{l}$. $\mathbb{S}^{i}$ denotes the interior of a simplex, and is given by removing all the proper faces from a simplex. A simplicial complex, denoted $\mathscr{M}$, is a collection of simplices such that $\mathbb{S}_{i} \in \mathscr{M}$ implies that all the faces of $\mathbb{S}_{i}$ are in $\mathscr{M}$, and the intersection of any two simplices is a face of both or empty. The local 
neighborhood of a vertex $\mathbf{x}_{j}$ can be defined in terms of its $\operatorname{star} \mathrm{S}\left(\mathbf{x}_{j}\right)$ which consists of all cofaces of $\mathbf{x}_{j}$. The star is not closed under taking faces.

A sampled vector field is given as a simplicial complex $\mathscr{M}$ with $m+1$ vertices $\left\{\mathbf{x}_{0}, \ldots, \mathbf{x}_{m}\right\}, \mathbf{x}_{i} \in \mathbb{R}^{n}$ and vector values $\left\{\mathbf{v}_{0}, \ldots, \mathbf{v}_{m}\right\}, \mathbf{v}_{i} \in \mathbb{R}^{n}$ defined at the vertices. The vector field is called generic when

1. $\left\|\mathbf{v}_{i}\right\|>0, \forall i \in\{0, \ldots, m\}$,

2. The vectors $\left\{\mathbf{v}_{i_{0}}, \ldots, \mathbf{v}_{i_{d}}\right\}$ at vertices $\left\{\mathbf{x}_{i_{0}}, \ldots, \mathbf{x}_{i_{d}}\right\}$ of the $d$-simplex $\mathbb{S}_{i} \in \mathscr{M}$ are affinely independent.

Note that not every vector field sampled from observations or simulations is generic, since the sampled vector magnitudes can be zero violating condition 1 . While we assume a generic vector field in the following discussion, Section 4.2 discusses how this assumption can be relaxed.

In this chapter, we focus on a class of interpolating vector valued functions which can be expressed as

$$
\mathbf{V}(\mathbf{x})=\sum_{\mathbb{S}_{i} \in \mathscr{M}} \mathbf{V}_{i}(\mathbf{x}), \quad \text { such that, } \quad \mathbf{V}_{i}(\mathbf{x})=\sum_{\mathbf{x}_{j} \in \mathbb{S}_{i}} w_{j}(\mathbf{x}) \mathbf{v}_{j}
$$

where, the weight functions $w_{j}(\mathbf{x})$ defined for vertices $\mathbf{x}_{j}$ are continuous, nonnegative, and local, meaning $w_{j}(\mathbf{x})>0, \forall \mathbf{x} \in \mathbf{S}\left(\mathbf{x}_{j}\right)$, and $w_{j}(\mathbf{x})=0, \forall \mathbf{x} \notin \mathbf{S}\left(\mathbf{x}_{j}\right)$.

Following the definition of the weight functions $w_{j}(\mathbf{x})$, it is clear that for the simplex $\mathbb{S}_{i}=\left\{\mathbf{x}_{i_{0}}, \ldots, \mathbf{x}_{i_{d}}\right\}, w_{j}(\mathbf{x})>0$ only for $j \in\left\{i_{0}, \ldots, i_{d}\right\}$. Furthermore, $w_{j}(\mathbf{x}) \rightarrow 0$ as $\mathbf{x} \rightarrow \mathbb{S}_{j}$, where $\mathbb{S}_{j}$ is a facet of $\mathbb{S}_{i}$. Since $\mathbf{V}_{i}$ are defined only on the interior of simplices, $\mathbf{V}$ is $C^{0}$ continuous across the faces of the simplices. It is simple to confirm that PL interpolation falls into this class of functions. Also, a variant of Radial Basis Function (RBF) interpolation falls into this class where the weights smoothly fall to zero at the boundary of the vertex stars.

\section{Critical Point Detection}

This section discusses how Brouwer degree theory can be used to robustly detect singularities in the class of interpolated vector fields defined in Section 3.2.

\subsection{Main Result}

Using the concepts introduced above we will show that a simplex $\mathbb{S}$ contains a critical point if and only if the origin, $\mathbf{0}$, lies in the convex hull of the vectors at the simplex's vertices. To connect the results of Section 3 with vector fields on simplices we define a one-to-one mapping from a simplex to an enclosing ball.

Let $\mathbb{S}=\left\{\mathbf{x}_{0}, \ldots, \mathbf{x}_{m}\right\}, m \leq n, \mathbf{x}_{i} \in \mathbb{R}^{n}$ be an $m$-simplex of $\mathscr{M}$. We can assume, without loss of generality, that the origin $\mathbf{0}$ is in the interior of $\mathbb{S}$. Let $\mathbb{B}$ be the unit ball. Let $\mathbf{x}(\neq \mathbf{0})$ be a point in the interior of $\mathbb{S}$, and $\mathbf{x}^{\prime}$ be the intersection of the ray from the origin through $\mathbf{x}$ with the boundary of $\mathbb{S}$. Then, we can define a mapping $\mathscr{B}: \mathbb{S} \rightarrow \mathbb{B}$ as $\mathscr{B}(\mathbf{x})=\mathbf{x} /\left\|\mathbf{x}^{\prime}\right\|$ for $\mathbf{x} \neq \mathbf{0}$, and $\mathscr{B}(\mathbf{0})=\mathbf{0}$. (see Fig. 3) 


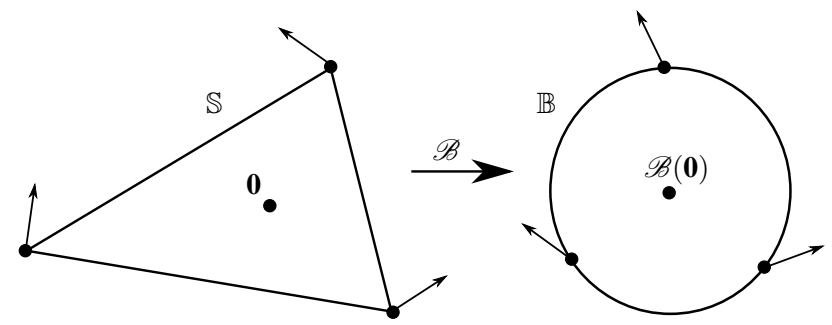

Fig. 3 The mapping $\mathscr{B}: \mathbb{S} \rightarrow \mathbb{B}$ in $\mathbb{R}^{2}$. The origin 0 is contained in the interior of $\mathbb{S}$.

Assuming non-degenerate simplices, $\mathscr{B}$ is continuous and invertible, with $\mathbf{0}$ as its fixed point, and we can use it to map $\mathbf{V}$ from any simplex in $\mathscr{M}$ onto an enclosing ball $\mathbb{B}$ with center in the interior. We now show that if $\mathbf{0}$ is contained in the convex hull of $\mathbf{V}$, the Brouwer degree of this simplex w.r.t. $\mathbf{0}$ is odd.

Lemma 1. Let $\mathbb{S}=\left\{\mathbf{x}_{0}, \ldots, \mathbf{x}_{n}\right\}, \mathbf{x}_{i} \in \mathbb{R}^{n}$ be a simplex of $\mathscr{M}$ containing the origin $\mathbf{0}$, and $\mathbf{V}$ be a vector field as defined above. If $\mathbf{0}$ lies in the convex hull of $\left(\mathbf{v}_{0}, \ldots, \mathbf{v}_{n}\right)$, then $\operatorname{deg}(\mathbf{V}, \mathbb{S}, \mathbf{0})$ is odd.

Proof. Let $\mathbb{B}$ be the unit ball of $\mathbb{S}$ as defined above. Furthermore, define $\overline{\mathbf{V}}: \mathbb{B} \rightarrow \mathbb{R}^{n}$, as $\overline{\mathbf{V}}(\mathbf{x})=\mathbf{V}\left(\mathscr{B}^{-1}(\mathbf{x})\right)$. Assume, there exists an $\mathbf{x}_{b} \in \partial \mathbb{B}$ such that

$$
\overline{\mathbf{V}}\left(\mathbf{x}_{b}\right)=a \cdot \overline{\mathbf{V}}\left(-\mathbf{x}_{b}\right), \text { with } a>0
$$

The following argumentation proves that this assumption leads to a contradiction.

Due to continuity of $\mathscr{B}$, it can not map adjacent points of $\mathbb{S}$ to antipodal points of $\mathbb{B}$, therefore it follows that there exist two different facets of $\mathbb{S}$, namely $\mathbb{S}_{j}$ and $\mathbb{S}_{k}$, containing parallel vectors. Let $\mathbf{x} \in \mathbb{S}_{j}$ and $\mathbf{y} \in \mathbb{S}_{k}$, such that for some $a>0$, $\sum_{i \neq j} w_{i}(\mathbf{x}) \mathbf{v}_{i}=a \cdot \sum_{i \neq k} w_{i}(\mathbf{y}) \mathbf{v}_{i}, j \neq k$.

$$
\begin{array}{r}
\sum_{i \neq j} w_{i}(\mathbf{x}) \mathbf{v}_{i}-a \cdot \sum_{i \neq k} w_{i}(\mathbf{y}) \mathbf{v}_{i}=0 \\
\sum_{i \neq j, k}\left(w_{i}(\mathbf{x})-a w_{i}(\mathbf{y})\right) \mathbf{v}_{i}-a w_{j}(\mathbf{y}) \mathbf{v}_{j}+w_{k}(\mathbf{x}) \mathbf{v}_{k}=0
\end{array}
$$

Also, since $\mathbf{0}$ lies in the convex hull of $\left(\mathbf{v}_{0}, \ldots, \mathbf{v}_{n}\right)$, we can find $c_{i}$ such that $\sum_{i=0}^{n} c_{i} \mathbf{v}_{i}=\mathbf{0}$, and $c_{i} \geq 0, \forall i$, and $\sum_{i=0}^{n} c_{i}=1$. Now, since $\left(\mathbf{v}_{0}, \ldots, \mathbf{v}_{n}\right)$ form an affine combination, $\sum \lambda_{i} \mathbf{v}_{i}=\sum \mu_{i} \mathbf{v}_{i} \Leftrightarrow \lambda_{i}=\mu_{i} \forall i$. This implies that

$$
\begin{aligned}
c_{i} & =w_{i}(\mathbf{x})-a w_{i}(\mathbf{y}) & \forall i \neq j, k \\
c_{k} & =w_{k}(\mathbf{x}) & \\
c_{j} & =-a w_{j}(\mathbf{y}) . &
\end{aligned}
$$

However, since all $c_{i}$ 's, $w_{i}$ 's, and $a$ are positive, the third condition gives a contradiction. Hence, a point $\mathbf{x}_{b}$ with $\overline{\mathbf{V}}\left(\mathbf{x}_{b}\right)=a \cdot \overline{\mathbf{V}}\left(-\mathbf{x}_{b}\right)$ does not exist. By Theorem 2, $\operatorname{deg}(\overline{\mathbf{V}}, \mathbb{B}, \mathbf{0})$, and hence $\operatorname{deg}(\mathbf{V}, \mathbb{S}, \mathbf{0})$ is odd. 
Combining all the results presented above allows us to prove the main result:

Theorem 3. Let $\mathbb{S}=\left\{\mathbf{x}_{0}, \ldots, \mathbf{x}_{n}\right\}, \mathbf{x}_{i} \in \mathbb{R}^{n}$ be a simplex of $\mathscr{M}$ and $\mathbf{V}$ be a vector field on $\mathscr{M}$ as defined above. Then $\mathbb{S}$ contains a critical point if and only if $\mathbf{0}$ is in the interior of the convex hull of $\mathbf{v}_{0}, \ldots, \mathbf{v}_{n}$.

Proof. If $\mathbf{0}$ is in the interior of the convex hull of $\mathbf{v}_{0}, \ldots, \mathbf{v}_{n}$, then the Brouwer degree of the origin inside $\mathbb{S}$ is odd (Lemma 1). By Theorem 1, there exists a critical point in $\mathbb{S}$.

If a critical point is located at $\mathbf{x}$ in $\mathbb{S}$ then

$$
\begin{array}{r}
\mathbf{v}(\mathbf{x})=\sum_{i=0}^{n} w_{i}(\mathbf{x}) \mathbf{v}_{i}=\mathbf{0} \\
\Rightarrow \sum_{i=0}^{n} \frac{w_{i}(\mathbf{x})}{\sum_{j=0}^{n} w_{j}(\mathbf{x})} \mathbf{v}_{i}=\sum_{i=0}^{n} c_{i}(\mathbf{x}) \mathbf{v}_{i}=\mathbf{0} .
\end{array}
$$

where, $c_{i}(\mathbf{x})=\frac{w_{i}(\mathbf{x})}{\sum_{j=0}^{n} w_{j}(\mathbf{x})}$. Since all the weights $w_{i}$ 's are non-negative, we have $0 \leq$ $c_{i} \leq 1, \forall i$, and $\sum_{i=0}^{n} c_{i}=1$. Hence, $\mathbf{0}$ is in the interior of the convex hull of $\mathbf{v}_{i}$ 's.

The practical implication of this result is that, to identify a simplex containing a critical point, one does not need to know the actual interpolation function as long as it satisfies the properties discussed in Section 3.2. Furthermore, it suffices to check the convex hull of the vectors at the vertices to find singularities. Thus, detection of singularities for any interpolation scheme is reduced to a simpler PL test which can be performed using the Simulation of Simplicity (SoS) [5] as will be discussed later.

Notice that if the space is sampled too sparsely, or a more complex interpolation scheme is used, multiple critical points may appear within a single simplex. In this case, our technique will assume the simplest possible interpretation of the vectors at the vertices. In particular, if the singularities in the same simplex cancel each other, then no singularities will be reported, since the vector field on the boundary can be completed with an entirely regular vector field in its interior.

The topological consistency of our technique can be proved as below.

Corollary 1. Using Theorem 3 always leads to topologically consistent critical point detection for $\mathbf{V}$ defined on $\mathscr{M}$ as above.

Proof. Given a closed orientable $n$-dimensional manifold $\mathbb{M}$, we consider its corresponding simplicial complex $\mathscr{M}$. We know that any smooth vector field on $\mathbb{M}$ must have an even number of critical points. Therefore, to demonstrate consistency, we show that our technique detects an even number of critical points for the sampled vector field $\mathbf{V}$ on $\mathscr{M}$.

We study $\mathscr{M}$ in function (vector) space by considering the simplicial complex defined by the vectors of $\mathbf{V}$, and denote it as $\mathscr{M}_{\mathbf{v}}$. Note that an orientable and closed $\mathscr{M}$ implies an orientable and closed $\mathscr{M}_{\mathbf{v}}$. There exists an embedding of $\mathscr{M}_{\mathbf{v}}$ in $\mathbb{R}^{n+1}$ where the $n+1^{\text {th }}$ component is chosen at random. We trace a line $\ell$ through the origin and orthogonal to the $\mathbb{R}^{n}$ subspace. Note that $\ell$ corresponds to zero vector 
0. By Theorem 3, singularities are not allowed to exist on the faces of simplices of $\mathscr{M}$. Since $\mathscr{M}_{\mathrm{v}}$ is closed, there exist an even number of transveral intersections between $\mathscr{M}_{\mathbf{v}}$ and $\ell$. Consequently, each intersection corresponds to a single simplex containing a critical point at its interior, leading to an even number of simplices with critical points; therefore, even number of critical points.

\subsection{Robust Computation using the Simulation of Simplicity (SoS)}

Detection of critical point in a simplex $\mathbb{S}$ requires a robust way of determining whether the zero vector is contained in the convex combination of the vectors of $\mathbb{S}$. Let $\mathbb{S}_{\mathbf{v}}$ denote the simplex created by the vectors of $\mathbf{V}$ at the vertices of $\mathbb{S}$, then detection of critical point simply translates into testing whether the origin $\mathbf{0}$ lies inside $\mathbb{S}_{\mathbf{v}}$. This point-in-simplex test can be further reduced to computing the orientation of the test point with respect to the facets of the simplex. This section describes how this procedure can be performed in a combinatorial manner.

The orientation of $n+1$ points in $n$-dimensional space can be computed as the sign of the following determinant

$$
\operatorname{det}\left(\mathbf{x}_{0}, \ldots, \mathbf{x}_{n}\right)=\left(\begin{array}{cccc}
\mathbf{x}_{0,0} & \ldots & \mathbf{x}_{0, n-1} & 1 \\
\vdots & \ddots & \vdots & 1 \\
\mathbf{x}_{n, 0} & \ldots & \mathbf{x}_{n, n-1} & 1
\end{array}\right)
$$

The determinant is zero if all the points lie on a common hyperplane. For example, in 2D, the orientation of three points is counter-clockwise (positive) if the sign of the corresponding determinant is positive, or clockwise (negative) if the sign is negative. The degenerate case where the three points are collinear leads to the determinant being zero. Recall that the sign of a determinant switches if an odd number of row-exchanges are carried out, therefore, care must be taken with respect to the order of the points. Algorithm 1 [5] calculates the orientation of a set of points, by first assigning (sorting) them a consistent order, and then computing the sign of the determinant.

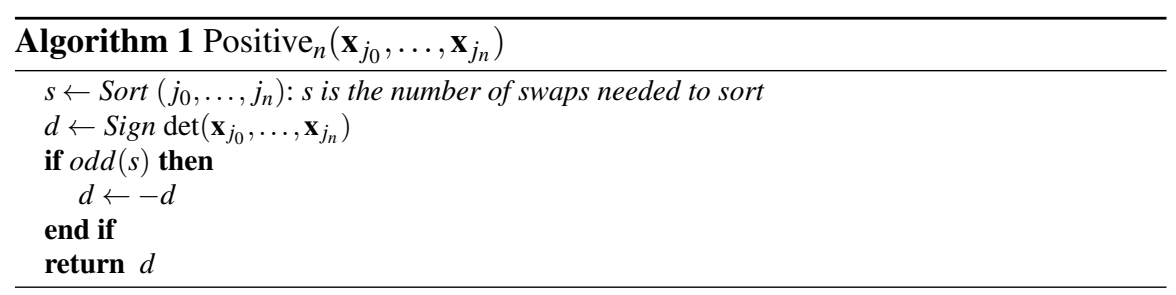

As discussed in Section 3.2, the determinant is always non-zero for generic vectors. To impose genericity, we use the Simulation of Simplicity ( $\mathrm{SoS}$ ) [5], which is a general-purpose programming technique to handle degeneracies in the data. The SoS applies a symbolic perturbation to the data preventing any of the determinants from becoming 0 , thus providing a non-degenerate point-in-simplex test. Intuitively, 
if a point lies on a shared coface of two or more simplices, the SoS makes a consistent choice by enforcing it to be contained inside one of them, while it lies outside the rest of them.

Numerical robustness is achieved by first converting the data to a fixed precision, and then representing the values as long integers in computing the determinant (as used in the Geomdir library [16]), hence removing the need for floating-point arithmetic. As a result, the determinant computation is both robust and combinatorial. We point out that Algorithm 1 is the fundamental step in the process of critical point detection, and helps achieve robustness by replacing numerical data (vector components) with combinatorial information (orientation).

Using Algorithm 1 for orientation computation, Algorithm 2 [5] performs the point-in-simplex test. Given a simplex $\mathbb{S}=\left\{\mathbf{x}_{0}, \ldots, \mathbf{x}_{n}\right\}, \mathbf{x}_{i} \in \mathbb{R}^{n}$, the first step is to determine the orientation of the facets of the simplex. Then, one by one each facet is replaced by the given test point and the corresponding orientation is computed. If all such combinations have the same orientation, then the test point lies inside the simplex, otherwise not.

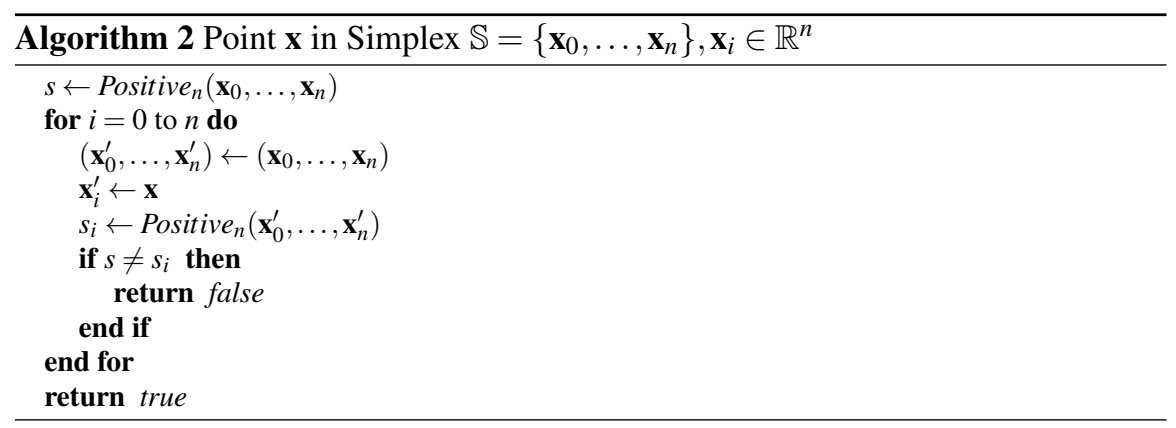

\section{Experimental Results}

We apply our technique to identify critical points in PL vector fields on a triangulated domain. For each simplex in the domain, we test for origin to lie inside the simplex created by the vectors at its vertices using SoS, and use the Geomdir library [16] to compute the determinants robustly. We compare our method with the numerical method to detect critical points [10], which solves a linear system for every simplex in the domain.

To evaluate the two techniques, we create a synthetic 2D vector fields with known critical points, two of which are carefully placed on an edge and a vertex. In Fig. 4, we see that for both of these critical points, the numerical technique determined that all the triangles sharing the corresponding edge and vertex respectively, contain critical points - a topologically inconsistent result. Our proposed method using SoS, however, makes a choice by representing each critical point as one triangle only, which matches the known ground truth.

To demonstrate dimension-independence of our technique, we test it on the Lorenz system (A 3D vector field $\mathbf{V}(x, y, z)=(\sigma(y-x), x(\rho-z)-y, x y-\beta z))$ 

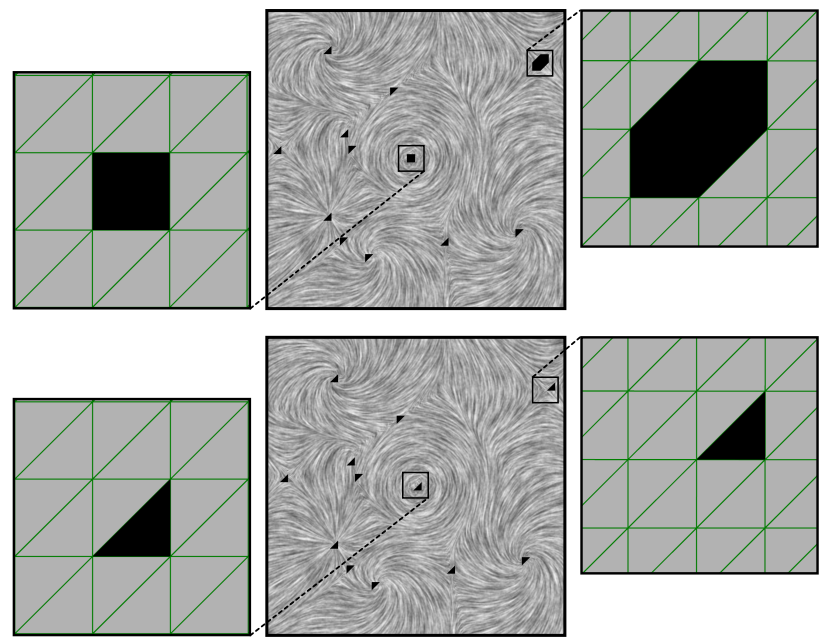

Fig. 4 Detection of singularities in 2D synthetic vector field. The top row shows the triangles where critical points were detected, numerically. Zoom-in views show that multiple triangles test positive for two critical points lying on the boundaries of triangles. Bottom row shows the consistent results detected using our algorithm where only one triangle per critical point tested positive.

with parameters $\sigma=10, \beta=8 / 3$, and $\rho=1 / 2$. For $\rho<1$, we expect the system to contain a singularity at the origin. Figure 5 shows the comparison where our technique selects a single tetrahedron touching the origin to represent the singularity. The numerical technique, on the other hand, detects all the 20 tetrahedra touching the origins as critical.

To compare the running times of the two methods, we test them on the highest slice of a 3D simulation of global ocean eddies [13], shown in Fig. 6. The ocean surface is represented by $10,633,760$ triangles. The numerical method [10] takes $\approx 89.22$ seconds to detect 24,552 critical points. On the other hand, our method takes only $\approx 6.85$ seconds to detect the same critical points, and thus, is significantly faster.

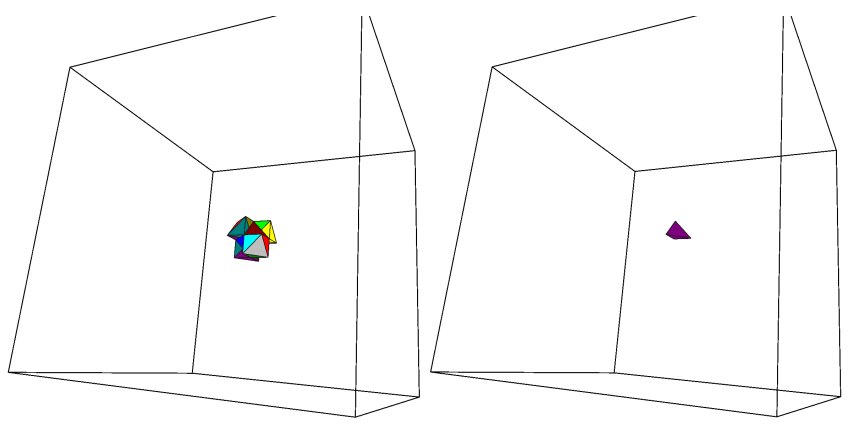

Fig. 5 Detection of singularities in the Lorenz system. Numerical method (left) detects 20 tets touching the origin, while our technique (right) detects a single tet to represent the singularity. 
Fig. 6 Detection of singularities in a 2D slice of simulation of global oceanic eddies [13]. The data contains 24,552 triangles with critical points, each represented by a black dot.

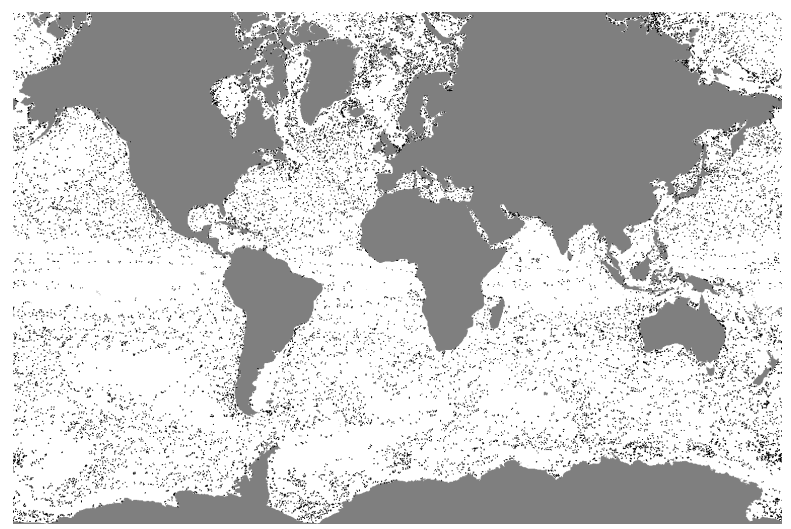

\section{Conclusions and Future Work}

In this chapter, we provide a necessary and sufficient condition for the existence of critical points in a simplex for a broad class of interpolated vector fields. Our existence condition for critical points allows us to develop a robust method to detect critical points in $n$-dimensions. Furthermore, when given finite precision values, the technique is guaranteed to use finite precision, and therefore the result can be computed exactly in a combinatorial manner. In the future, we wish to investigate further the class of interpolation functions we showed this result for, identifying which other interpolation techniques fall into this class. We also wish to extend this work to include classification of critical points.

Acknowledgements We thank Mathew Maltude from the Climate, Ocean and Sea Ice Modelling program at Los Alamos National Laboratory and the BER Office of Science UV-CDAT team for providing us access to the ocean data from Fig. 6. This work was supported in part by NSF OCI-0906379, NSF OCI-0904631, DOE/NEUP 120341, DOE/MAPD DESC000192, DOE/LLNL B597476, DOE/Codesign P01180734, and DOE/SciDAC DESC0007446. This work was also performed under the auspices of the US Department of Energy (DOE) by Lawrence Livermore National Laboratory (LLNL) under contract DE-AC52-07NA27344. LLNL-CONF-xxxxx.

\section{References}

1. R. Batra and L. Hesselink. Feature comparisons of 3-D vector fields using earth mover's distance. In Proc. of IEEE Vis., pages 105-114, 1999.

2. P.-T. Bremer, G. Weber, V. Pascucci, M. Day, and J. Bell. Analyzing and tracking burning structures in lean premixed hydrogen flames. IEEE Trans. on Vis. and Comp. Graph., 16(2):248-260, 2010

3. G. Chen, K. Mischaikow, R. S. Laramee, P. Pilarczyk, and E. Zhang. Vector field editing and periodic orbit extraction using Morse decomposition. IEEE Trans. on Vis. and Comp. Graph., 13(4):769-785, 2007.

4. G. Chen, K. Mischaikow, R. S. Laramee, and E. Zhang. Efficient Morse decompositions of vector fields. IEEE Trans. on Vis. and Comp. Graph., 14(4):848-862, 2008.

5. H. Edelsbrunner and E. P. Mücke. Simulation of simplicity: A technique to cope with degenerate cases in geometric algorithms. ACM Trans. on Graph., 9:66-104, 1990.

6. G. Elber and M.-S. Kim. Geometric constraint solver using multivariate rational spline functions. In Proc. of ACM Symp. on Solid Modeling and Apps. (SMA '01), pages 1-10, 2001. 
7. C. Garth, X. Tricoche, and G. Scheuermann. Tracking of vector field singularities in unstructured 3D time-dependent datasets. In Proc. of IEEE Vis., pages 329-336, 2004.

8. J. L. Helman and L. Hesselink. Representation and display of vector field topology in fluid flow data sets. IEEE Computer, 22(8):27-36, 1989.

9. D. Laney, P.-T. Bremer, A. Mascarenhas, P. Miller, and V. Pascucci. Understanding the structure of the turbulent mixing layer in hydrodynamic instabilities. IEEE Trans. on Vis. and Comp. Graph, 12(5):1052-1060, 2006.

10. Y. Lavin, R. Batra, and L. Hesselink. Feature comparisons of vector fields using earth mover's distance. In Proc. of IEEE Vis., pages 103-109, 1998.

11. W.-C. Li, B. Vallet, N. Ray, and B. Levy. Representing higher-order singularities in vector fields on piecewise linear surfaces. IEEE Trans. on Vis. and Comp. Graph., 12(5), 2006.

12. N. G. Lloyd. Degree theory. Cambridge University Press, 1978.

13. M. Maltrud, F. Bryan, and S. Peacock. Boundary impulse response functions in a century-long eddying global ocean simulation. Environmental Fluid Mechanics, 10:275-295, 2010.

14. S. Mann and A. Rockwood. Computing singularities of $3 \mathrm{~d}$ vector fields with geometric algebra. In Proc. of IEEE Vis., pages 283-290, 2002.

15. A. Mascarenhas, R. W. Grout, P.-T. Bremer, E. R. Hawkes, V. Pascucci, and J. Chen. Topological feature extraction for comparison of terascale combustion simulation data. In Topological Methods in Data Analysis and Vis. II, Mathematics and Vis., pages 229-240, 2011.

16. E. P. Mucke. Geomdir - http://www.geom.uiuc.edu/software/cglist/GeomDir/.

17. K. Polthier and E. Preuß. Identifying vector field singularities using a discrete Hodge decomposition. In H. Hege and K. Polthier, editors, Mathematical Vis. III, pages 112-134, 2003.

18. J. Reininghaus and I. Hotz. Combinatorial 2D vector field topology extraction and simplification. In Topological Methods in Data Analysis and Vis. Theory, Algorithms, and Apps. Springer, 2009

19. J. Reininghaus, C. Löwen, and I. Hotz. Fast combinatorial vector field topology. IEEE Trans. on Vis. and Comp. Graph., 17:1433-1443, 2011.

20. G. Scheuermann, H. Krüger, M. Menzel, and A. P. Rockwood. Visualizing nonlinear vector field topology. IEEE Trans. on Vis. and Comp. Graph., 4(2):109-116, 1998.

21. G. Scheuermann and X. Tricoche. Topological methods for flow visualization. In C. D. Hansen and C. R. Johnson, editors, The Visualization Handbook, pages 341-356. Elsevier, 2005.

22. S. Schirra. Robustness and precision issues in geometric computation. In J.-R. Sack and J. Urrutia, editors, Handbook of Computational Geometry, chapter 14, Elsevier, 2000.

23. H. Theisel, C. Rössl, and H.-P. Seidel. Compression of 2D vector fields under guaranteed topology preservation. Comp. Graph. Forum (Proc. Eurographics), 22(3), 2003.

24. H. Theisel, T. Weinkauf, H.-C. Hege, and H.-P. Seidel. Saddle connectors - an approach to visualizing the topological skeleton of complex 3D vector fields. In Proc. of IEEE Vis., 2003.

25. X. Tricoche, C. Garth, and A. Sanderson. Visualization of topological structures in area preserving maps. IEEE Trans. on Vis. and Comp. Graph., 17(12):1765-1774, 2011.

26. X. Tricoche, C. Garth, A. Sanderson, and K. Joy. Visualizing invariant manifolds in areapreserving maps. In Topological Methods in Data Analysis and Vis. II, Mathematics and Vis., pages 109-124, 2011.

27. X. Tricoche, G. Scheuermann, and H. Hagen. Higher order singularities in piecewise linear vector fields. In The Mathematics of Surfaces IX, pages 99-113. Springer, 2000.

28. X. Tricoche, G. Scheuermann, and H. Hagen. A topology simplification method for 2D vector fields. In Proc. of IEEE Vis., pages 359-366, 2000.

29. X. Tricoche, G. Scheuermann, and H. Hagen. Continuous topology simplification of planar vector fields. In Proc. of IEEE Vis., pages 159-166, 2001.

30. T. Weinkauf, H. Theisel, H.-C. Hege, and H.-P. Seidel. Toplogical construction and visualization of higher order 3D vector fields. Comp. Graph. Forum (Proc. Eurographics), 23(3), 2004.

31. T. Weinkauf, H. Theisel, K. Shi, H.-C. Hege, and H.-P. Seidel. Extracting higher order critical points and topological simplification of 3D vector fields. In Proc. of IEEE Vis., 2005.

32. C. K. Yap. Robust geometric computation. In J. E. Goodman and J. O'Rourke, editors, Handbook of Discrete and Computational Geometry, chapter 41, pages 927-952. Chapmen \& Hall, 2004. 